Research Paper

\title{
Prognostic Value of Plasma Fibrinogen in Lung Cancer Patients: A Meta-Analysis
}

\author{
Hai Zhong, Yingying Qian, Surong Fang, Ying Wang, Yun Tang, Wei Gu ${ }^{\bowtie}$ \\ Department of Respiration, Nanjing First Hospital, Nanjing Medical University, Nanjing, People's Republic of China. \\ $\triangle$ Corresponding author: Wei Gu, Department of Respiration, Nanjing First Hospital, Nanjing Medical University, 68 Changle Road, Nanjing 210006, Jiangsu \\ Province, People's Republic of China. Tel: + 8625 87726380; E-mail: guwei@njmu.edu.cn. \\ (c) Ivyspring International Publisher. This is an open access article distributed under the terms of the Creative Commons Attribution (CC BY-NC) license \\ (https://creativecommons.org/licenses/by-nc/4.0/). See http://ivyspring.com/terms for full terms and conditions.
}

Received: 2018.03.28; Accepted: 2018.08.17; Published: 2018.10.10

\begin{abstract}
Background: The prognostic role of plasma fibrinogen in lung cancer remains controversial. The aim of this meta-analysis was to assess the prognostic value of plasma fibrinogen in lung cancer.

Methods: We performed a systematic literature search to identify eligible studies in PubMed, Embase and the Cochrane Library database. The hazard ratios (HR) and their $95 \%$ confidence intervals $(\mathrm{Cl})$ were collected from these eligible studies and were used to assess the relationship between plasma fibrinogen and lung cancer.

Results: A total of 16 studies including 6,881 patients were selected in this meta-analysis. The results showed that elevated plasma fibrinogen in lung cancer patients was correlated with poor overall survival (OS) $(\mathrm{HR}=1.38,95 \% \mathrm{Cl}$ : 1.22-1.55, $\mathrm{P}<0.001)$ and disease-free survival (DFS) / progress-free survival (PFS). (HR = 1.29, 95\% Cl: 1.01-1.65, $\mathrm{P}=0.042)$. When stratified by cut-off value for OS and DFS/PFS, there was no significant heterogeneity. And the results of "cut-off value $\geq 400 \mathrm{mg} / \mathrm{dl}$ " group showed that the high level of fibrinogen in serum was associated with worse OS and DFS/PFS of lung cancer. In further subgroup analysis by tumor histology, high plasma fibrinogen was also associated with worse OS in non-small cell lung cancer (NSCLC) $(\mathrm{HR}=1.32,95 \% \mathrm{Cl}$ : 1.14-1.53, $\mathrm{P}<0.001)$. However, there was no significant association between high plasma fibrinogen and poor DFS in NSCLC patients $(\mathrm{HR}=1.24,95 \% \mathrm{Cl}$ : 0.97-1.57, $\mathrm{P}=0.08)$. The Egger's regression test indicated evidence of publication bias for DFS/PFS.

Conclusions: Elevated plasma fibrinogen, particularly defined as a plasma fibrinogen concentration of $\geq 400 \mathrm{mg} / \mathrm{dl}$, could be a promising indicator for worse OS in lung cancer patients, including NSCLC.
\end{abstract}

Key words: Fibrinogen, Lung cancer, Non-small cell lung cancer, Prognosis, Meta-analysis

\section{Introduction}

Lung cancer, which threatens seriously human life and health, remains the leading cause of cancer-related death in the world [1]. Despite the development of molecular targeted therapy and improvements of chemoradiotherapy in lung cancer, the prognosis of lung cancer remains poor, with the 5-year survival rate has not improved significantly [2]. Currently several nonspecific tumor markers, such as carcinoembryonic antigen (CEA), cytokeratin 19 fragment (CYFRA 21-1) and neuron-specific-enolase (NSE) have been used to investigate the prognostic value of lung cancer, however, clinical studies have produced conflicting results, and reliable markers are still lacking [3-7].

In recent studies, many researchers have found the relationship between blood coagulation disorders and several malignant tumors $[8,9]$. Activation of the coagulation system and procoagulant changes is correlated with tumor invasion, progression, angiogenesis and metastasis [8-12]. Fibrinogen, as a plasma coagulation factor synthesized by the liver, can reflect the degree of activation of coagulation 
system by its high levels. Elevated plasma fibrinogen levels are associated with poor outcomes in a variety of malignant tumors [13]. Moreover, the prognostic role of plasma fibrinogen in lung cancer patients has been investigated in several published studies. However, the results remain inconclusive [14-17]. Therefore, we performed this meta-analysis to investigate the correlation between elevated plasma fibrinogen and prognosis in patients with lung cancer.

\section{Materials and Methods}

\section{Search strategy and study selection}

Relevant literature in the period from inception up to February 2018 was systematically searched using PubMed, Embase and the Cochrane Library database. The key search items used were as follows: (fibrinogen or "plasma fibrinogen") and ("lung carcinoma" or "lung cancer" or "lung tumor" or "lung neoplasm") and (prognosis or prognostic or survival or outcome). In order not to omit the potentially relevant articles, reference lists in the retrieved papers were also screened.

In this meta-analysis, eligible studies were included according to the following criteria: (1) any clinical study comprising the association between plasma fibrinogen and prognosis of lung cancer patients was eligible; (2) data of hazard ratios (HR) and $95 \%$ confidence intervals $(\mathrm{CI})$ were provided, or sufficient information that allowed manual calculation of them; (3) the studies were published only in English; (4) for studies with overlapping data, we selected the most complete or the latest study. The studies with the following criteria were excluded: (1) studies that were published as letters, reviews, case reports and conference abstracts; (2) overlapping studies or duplicated data; (3) full text unavailable and non-English article; (4) studies lacking sufficient data to acquire HR and their 95\% CI.

\section{Data extraction and quality assessment}

In order to reduce the risk of losing a publication, two investigators (Hai Zhong and Yingying Qian) independently surveyed the eligible literatures based on the predefined criteria. In addition, any inconsistent opinions between the two investigators were resolved by consensus involving a third reviewer (Surong Fang). Data were extracted from all eligible studies as follow: name of the first author, year of publication, study country, number of patient enrolled, pathological type, clinical stages, cut-off value, $\mathrm{HR}$ and their $95 \% \mathrm{CI}$ for overall survival (OS) and disease-free survival (DFS) / progress-free survival (PFS).

The two investigators independently evaluated the quality of each included study using the Newcastle-Ottawa scale (NOS) for cohort studies [18]. The three major components of NOS criteria were: selection of cohorts (0-4 points), comparability of cohorts (0-2 points), ascertainment of outcomes (0-3 points). Studies with NOS scores of more than 6 were considered as high quality.

\section{Statistical analysis}

$\mathrm{HR}$ and 95\% CI were used to analyse the prognostic value of elevated plasma fibrinogen in lung cancer patients. Meta-analysis was conducted to merge the results of the included studies. The combined HR $>1$ and its $95 \%$ CI did not overlap 1 indicated that a higher level of plasma fibrinogen could be a poorer prognostic factor in lung cancer patients, whereas the combined HR $<1$ indicated better prognosis. Heterogeneity among the included studies was assessed using Cochrane's $Q$ test and $\mathrm{I}^{2}$ statistic [19]. A P-value $<0.10$ and/or $\mathrm{I}^{2}>50 \%$ indicated significant heterogeneity among studies and a random effects was used to calculate the pooled HR and $95 \%$ CI. Otherwise, the fixed effects model was adopted [20, 21]. The potential for publication bias was evaluated using the Begg's test and Egger's regression test, and a probability value less than 0.05 was used to indicate a statistically significant difference [22, 23]. Moreover, a sensitivity analysis was conducted to validate the stability of the pooled results by omitting individual study [24]. All data analyses were performed using the STATA 12.0 software.

\section{Results}

\section{Study selection and characteristics}

The detailed flow diagram for the selection of eligible studies is shown in Figure 1. A total of 459 related records were identified through literature searching according to the abovementioned criteria. After screening the titles and abstracts, 424 independent studies were excluded. The full texts of the remaining 35 studies were further evaluated. Finally, only 16 studies were included in this meta-analysis [14-17, 25-36].

The basic features of the included studies are summarized in Table 1. As presented in Table 1, there were 6,881 lung cancer patients from 4 different countries included in our meta-analysis, with publication year ranging from 1997 to 2017 . There were 10 studies included non-small cell lung cancer (NSCLC) patients, only 2 studies included small cell lung cancer (SCLC), and the other 4 studies included all types of lung cancer patients. Among the 16 studies, 15 studies investigated the relationship 
between elevated plasma fibrinogen and OS of lung cancer, and the association between elevated plasma fibrinogen and DFS/PFS was evaluated only in 7 studies. A total of 15 studies reported HR and 95\% CI. And for the remaining one study [25], we calculated the data of $\mathrm{HR}$ and $95 \%$ CI using available information based on the methods provided in the literature by Parmar et al [37]. The quality assessment of each included study using the NOS is demonstrated in Table 2.

\section{Relationship between plasma fibrinogen and OS of lung cancer}

Among the eligible studies, 15 studies including 6,697 patients had assessed the relationship between plasma fibrinogen and OS of lung cancer. Because of significant heterogeneity $\left(\mathrm{P}=0.004, \mathrm{I}^{2}=56.6 \%\right)$, we used a random effect model to calculate the pooled HR. The pooled results indicated a significantly shorter OS in lung cancer patients with high plasma fibrinogen compared to those with low plasma fibrinogen $(\mathrm{HR}=1.38,95 \% \mathrm{CI}: 1.22-1.55,<0.001$; Figure 2A). Then, we performed subgroup analysis according to six potential clinical variables, including analysis of variable, ethnicity, cut-off value, tumor stage, histology, and quality score.

As demonstrated in Table 3, stratified analysis by analysis of variable revealed that high plasma fibrinogen was associated with worse OS of lung cancer patients both in univariate analysis $(\mathrm{HR}=1.65$, 95\% CI: 1.45-1.87, P < 0.001) and multivariate analysis $(\mathrm{HR}=1.35,95 \% \mathrm{CI}: 1.19-1.54, \mathrm{P}<0.001)$. Subgroup analyses by ethnicity, we found the pooled HR was 1.36 (95\% CI: $1.19-1.55)$ for Asian patients and 1.48 (95\% CI: $1.22-1.81)$ for non-Asian patients. Stratification by cut-off value, the data indicated that the pooled HR was 1.23 (95\% CI: 1.09-1.38) for "cut-off value $<400 \mathrm{mg} / \mathrm{dl}^{\prime \prime}$ group and 1.48 (95\% CI: 1.34-1.64) for "cut-off value $\geq 400 \mathrm{mg} / \mathrm{dl}$ " group. Notably, there were no significant heterogeneity both in "cut-off value $<400 \mathrm{mg} / \mathrm{dl}^{\prime \prime}$ group $\left(\mathrm{P}=0.226, \mathrm{I}^{2}=29.30 \%\right)$ and "cut-off value $\geq 400 \mathrm{mg} / \mathrm{dl}^{\prime}$ group $\left(\mathrm{P}=0.616, \mathrm{I}^{2}=\right.$ $0.00 \%)$. Similarly, subgroup analyses showed that the elevated plasma fibrinogen was a poor prognostic marker of OS regardless of tumor histology and NOS score. In addition, stratified analysis by tumor stage showed high fibrinogen was associated with poor OS in "III-IV stage" group (HR $=1.41,95 \%$ CI: 1.22-1.62, P $<0.001)$, while there was no statistically significant association in "I-III stage" group (HR $=1.24,95 \% \mathrm{CI}$ : 0.99-1.55, $\mathrm{P}=0.058$ ).

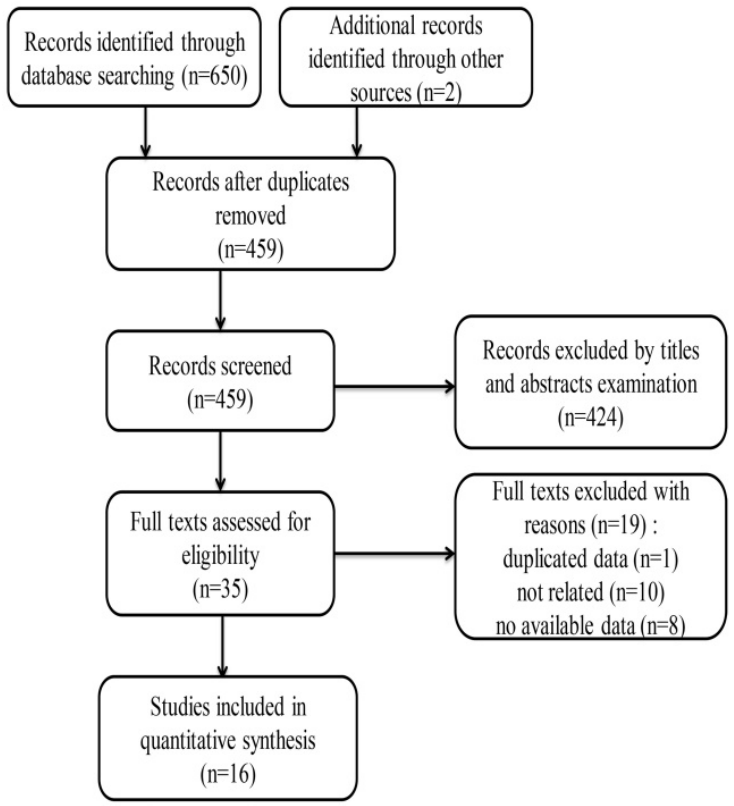

Figure 1. Flow chart of search strategy and study selection.

Table 1. Main characteristics of the included studies in our meta-analysis

\begin{tabular}{|c|c|c|c|c|c|c|c|c|c|c|}
\hline \multirow[t]{2}{*}{ Study/Year } & \multirow{2}{*}{ Country } & \multirow{2}{*}{$\begin{array}{l}\text { Patient } \\
\text { number }\end{array}$} & \multirow{2}{*}{ Histology } & \multirow[t]{2}{*}{ Stage } & \multirow{2}{*}{$\begin{array}{l}\text { Cut-off } \\
\text { value }\end{array}$} & \multirow[t]{2}{*}{ Outcome } & \multicolumn{2}{|l|}{ OS: HR(95\%CI) } & \multicolumn{2}{|c|}{ DFS/PFS: HR(95\%CI) } \\
\hline & & & & & & & $\mathrm{U}$ & $\mathbf{M}$ & $\mathbf{U}$ & $\mathbf{M}$ \\
\hline Buccheri G/ 1997 & France & 247 & NSCLC+SLCL & NR & 429 & OS & $1.46(1.16-1.84)$ & NR & NR & NR \\
\hline Unsal E/ 2004 & Turkey & 58 & NSCLC+SLCL & I-IV & 350 & OS & NR & $1.28(0.73-2.23)$ & NR & NR \\
\hline Altiay G/ 2007 & Turkey & 78 & NSCLC+SLCL & III-IV & 380 & OS & $1.86(1.08-3.21)$ & NR & NR & NR \\
\hline Zhao J/ 2012 & China & 160 & NSCLC & III-IV & 440 & OS & NR & $1.673(1.133-2.471)$ & NR & NR \\
\hline Sheng L/ 2013 & China & 567 & NSCLC & I-IIIB & 400 & OS/DFS & $2.07(1.37-3.14)$ & $1.64(1.06-2.53)$ & $1.57(1.15-2.13)$ & $1.49(1.07-2.05)$ \\
\hline Zhu JF/ 2014 & China & 275 & NSCLC & IV & 400 & OS & NR & $1.5(1.1-2.1)$ & NR & NR \\
\hline Kim KH/ 2014 & Korea & 854 & NSCLC & III-IV & 450 & OS & NR & $1.34(1.13-1.58)$ & NR & NR \\
\hline Jiang HG/ 2014 & China & 184 & NSCLC & I-IIIA & 400 & DFS & NR & NR & NR & $3.36(1.19-9.26)$ \\
\hline Chen YS/ 2014 & China & 370 & NSCLC+SLCL & I-IV & 420 & OS & NR & $1.497(1.115-2.008)$ & NR & NR \\
\hline Zhang H/ 2015 & China & 1238 & NSCLC & I-IIIA & NR & OS/DFS & $1.370(1.180-1.592)$ & $0.935(0.778-1.119)$ & $1.335(1.149-1.550)$ & $0.871(0.730-1.053)$ \\
\hline Zhu LR/ 2016 & China & 74 & SCLC & - & 400 & 0S/PFS & NR & $3.66(1.10-12.57)$ & NR & $3.62(1.03-12.32)$ \\
\hline Zeng Q/ 2017 & China & 856 & NSCLC & I-IV & 370 & OS & $2.064(1.761-2.420)$ & $1.399(1.122-1.744)$ & NR & NR \\
\hline Fan S/ 2017 & China & 120 & SCLC & - & 400 & OS & $1.591(1.085-2.332)$ & $1.505(1.018-2.226)$ & NR & NR \\
\hline Liu W/ 2017 & China & 1120 & NSCLC & I-IIIA & 360 & OS/DFS & $1.451(1.242-1.695)$ & $1.079(0.904-1.287)$ & $1.405(1.203-1.641)$ & $1.024(0.859-1.221)$ \\
\hline Gao L/ 2017 & China & 546 & NSCLC & I-IIIA & 354 & 0S/DFS & $1.580(1.257-1.985)$ & $1.217(0.954-1.554)$ & $1.559(1.241-1.960)$ & $1.164(0.910-1.490)$ \\
\hline Wang YQ/ 2017 & China & 134 & NSCLC & I-IIIA & 400 & 0S/DFS & $2.131(1.417-3.206)$ & $2.045(1.332-3.141)$ & $1.875(1.202-2.925)$ & $1.865(1.196-2.910)$ \\
\hline
\end{tabular}


Table 2. Quality assessment of included studies using the Newcastle-Ottawa Scale

\begin{tabular}{lllll}
\hline Study & Selection & Comparability & Outcome & Total score \\
\hline Buccheri G & 4 & 1 & 3 & 8 \\
Unsal E & 4 & 0 & 2 & 6 \\
Altiay G & 4 & 0 & 2 & 6 \\
Zhao J & 4 & 1 & 2 & 7 \\
Sheng L & 4 & 1 & 2 & 7 \\
Zhu JF & 4 & 1 & 2 & 7 \\
Kim KH & 4 & 0 & 2 & 6 \\
Jiang HG & 4 & 0 & 2 & 6 \\
Chen YS & 4 & 1 & 2 & 7 \\
Zhang H & 4 & 0 & 3 & 7 \\
Zhu LR & 4 & 1 & 2 & 7 \\
Zeng Q & 4 & 1 & 2 & 7 \\
Fan S & 4 & 0 & 3 & 7 \\
Liu W & 4 & 0 & 3 & 7 \\
Gao L & 4 & 0 & 2 & 6 \\
Wang YQ & 4 & 0 & 2 & 6
\end{tabular}

\section{Relationship between plasma fibrinogen and} DFS/PFS of lung cancer

Only 7 studies with 3,863 cases presented the data of high plasma fibrinogen and DFS/PFS in lung cancer patients. The random effect model was applied owing to significant heterogeneity was tested $(\mathrm{P}=$ $0.001, \mathrm{I}^{2}=74.90 \%$ ), and the results of the pooled HR demonstrated that high plasma fibrinogen was correlated with worse DFS/PFS in lung cancer (HR = 1.29, 95\% CI: 1.01-1.65, < 0.001; Figure 2B).

As shown in Table 3, subgroup analysis revealed that elevated plasma fibrinogen was associated with unfavorable DFS/PFS of lung cancer both in univariate analysis ( $\mathrm{HR}=1.43,95 \% \mathrm{CI}: 1.31-1.57, \mathrm{P}<$ 0.001 ) and multivariate analysis ( $\mathrm{HR}=1.29,95 \% \mathrm{CI}$ : 1.01-1.65, $\mathrm{P}=0.042$ ). Stratified analysis according to ethnicity was not performed due to all the people included in the studies was Asian. In addition, elevated fibrinogen predicted poor DFS/PFS for patients in "cut-off value $\geq 400 \mathrm{mg} / \mathrm{dl}$ " group. The results of remaining groups including "cut-off value < $400 \mathrm{mg} / \mathrm{dl}$ " group, "I-III stage" group, "NSCLC" group, low quality studies group (NOS $\leq 6)$ and high quality studies group (NOS $>6$ ) showed no significant association between high plasma fibrinogen and DFS/PFS.

Table 3. The results of stratified analysis in meta-analysis for OS and DFS/PFS

\begin{tabular}{|c|c|c|c|c|c|c|c|}
\hline \multirow[t]{2}{*}{ Outcome } & \multirow[t]{2}{*}{ Stratified analysis } & \multirow[t]{2}{*}{ No. of studies } & \multirow[t]{2}{*}{ HR $(95 \%$ CI $)$} & \multirow[t]{2}{*}{$\mathbf{P}$} & \multicolumn{2}{|c|}{ Heterogeneity } & \multirow[t]{2}{*}{ Model used } \\
\hline & & & & & $\mathrm{I}^{2}(\%)$ & $\mathbf{P}_{\mathbf{h}}$ & \\
\hline \multirow[t]{19}{*}{ OS } & Analysis of variable & & & & & & \\
\hline & Univariate & 9 & $1.65(1.45-1.87)$ & $<0.001$ & $59.50 \%$ & 0.011 & Random \\
\hline & Multivariate & 13 & $1.35(1.19-1.54)$ & $<0.001$ & $59.00 \%$ & 0.004 & Random \\
\hline & Ethnicity & & & & & & \\
\hline & Asian & 12 & $1.36(1.19-1.55)$ & $<0.001$ & $62.40 \%$ & 0.002 & Random \\
\hline & non-Asian & 3 & $1.48(1.22-1.81)$ & $<0.001$ & $0.00 \%$ & 0.622 & Fixed \\
\hline & Cut-off value & & & & & & \\
\hline & $<400 \mathrm{mg} / \mathrm{dl}$ & 5 & $1.23(1.09-1.38)$ & $<0.001$ & $29.30 \%$ & 0.226 & Fixed \\
\hline & $\geq 400 \mathrm{mg} / \mathrm{dl}$ & 9 & $1.48(1.34-1.64)$ & $<0.001$ & $0.00 \%$ & 0.616 & Fixed \\
\hline & Tumor stage & & & & & & \\
\hline & I-III & 5 & $1.24(0.99-1.55)$ & 0.058 & $73.20 \%$ & 0.005 & Random \\
\hline & III-IV & 3 & $1.41(1.22-1.62)$ & $<0.001$ & $0.00 \%$ & 0.54 & Fixed \\
\hline & Histology & & & & & & \\
\hline & NSCLC & 9 & $1.32(1.14-1.53)$ & $<0.001$ & $66.70 \%$ & 0.002 & Random \\
\hline & SCLC & 2 & $1.64(1.13-2.37)$ & 0.01 & $46.10 \%$ & 0.173 & Fixed \\
\hline & NSCLC+SCLC & 4 & $1.49(1.26-1.75)$ & $<0.001$ & $0.00 \%$ & 0.813 & Fixed \\
\hline & Quality score & & & & & & \\
\hline & $>6$ & 10 & $1.36(1.17-1.59)$ & $<0.001$ & $64.50 \%$ & 0.003 & Random \\
\hline & $\leq 6$ & 5 & $1.37(1.21-1.56)$ & $<0.001$ & $28.40 \%$ & 0.232 & Fixed \\
\hline \multirow[t]{14}{*}{ DFS/PFS } & Analysis of variable & & & & & & \\
\hline & Univariate & 5 & $1.43(1.31-1.57)$ & $<0.001$ & $0.00 \%$ & 0.527 & Fixed \\
\hline & Multivariate & 7 & $1.29(1.01-1.65)$ & 0.042 & $74.90 \%$ & 0.001 & Random \\
\hline & Cut-off value & & & & & & \\
\hline & $<400 \mathrm{mg} / \mathrm{dl}$ & 2 & $1.07(0.93-1.23)$ & 0.36 & $0.00 \%$ & 0.407 & Fixed \\
\hline & $\geq 400 \mathrm{mg} / \mathrm{dl}$ & 4 & $1.74(1.36-2.23)$ & $<0.001$ & $22.80 \%$ & 0.247 & Fixed \\
\hline & Tumor stage & & & & & & \\
\hline & I-III & 6 & $1.24(0.97-1.57)$ & 0.08 & $75.30 \%$ & 0.001 & Random \\
\hline & Histology & & & & & & \\
\hline & NSCLC & 6 & $1.24(0.97-1.57)$ & 0.08 & $75.30 \%$ & 0.001 & Random \\
\hline & SCLC & 1 & - & - & - & - & - \\
\hline & Quality score & & & & & & \\
\hline & $>6$ & 4 & $1.13(0.85-1.51)$ & 0.387 & $75.20 \%$ & 0.007 & Random \\
\hline & $\leq 6$ & 3 & $1.65(1.00-2.73)$ & 0.051 & $69.00 \%$ & 0.04 & Random \\
\hline
\end{tabular}

OS: overall survival; DFS: disease-free survival; PFS: progress-free survival; NSCLC: non-small cell lung cancer; SCLC: small cell lung cancer; HR: hazard ratio; CI: confidence interval 


\section{Publication bias}

The potential for publication bias was detected by Begg's test and Egger's regression test. Visual inspection of the Begg's funnel plot demonstrated evidence of publication bias for OS (Figure 3A), which was confirmed by Begg's test $(P=0.013)$ and Egger's regression test $(P=0.004)$. Then we adopted the trim and fill method to adjust the asymmetry of the funnel plot (Figure 3B). The pooled analysis included the hypothetical studies continued to indicate a statistically significant association between high plasma fibrinogen and OS $(\mathrm{HR}=1.26,95 \% \mathrm{CI}$ : 1.12-1.41, $\mathrm{P}<0.001)$. The result showed that the effect

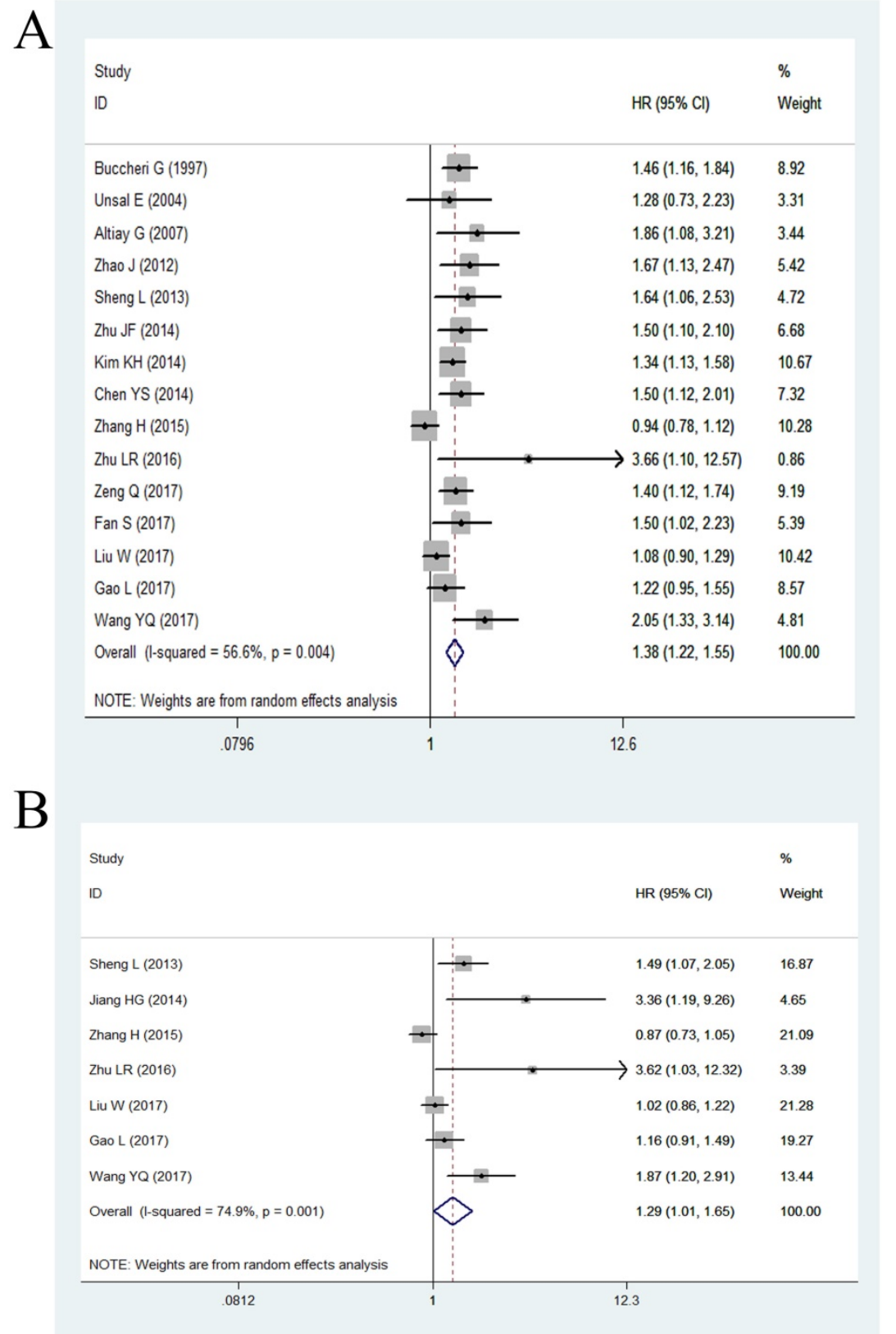

Figure 2. Forest plot showing the pooled HR for the correlation between elevated plasma fibrinogen and OS (A) or DFS/PFS (B) in lung cancer patients. of publication bias was not significant and the conclusion was relatively stable.

We also used the Begg's test and Egger's regression test to evaluated publication bias for DFS/PFS. The Begg's funnel plot funnel plots revealed a low probability of publication bias for DFS/PFS (Figure 3C). Although the Begg's test ( $\mathrm{P}=$ 0.133) was not statisitically significant, the Egger's regression test $(\mathrm{P}=0.007)$ indicated evidence of publication bias for DFS/PFS. The trim and fill method was used to adjust for publication bias under the random-effects model (Figure 3D), and the corrected pooled HR for DFS/PFS was 1.08 (95\% CI: $0.83-1.40, \mathrm{P}=0.592)$. These indicated that there could be publication bias for DFS/PFS, and the conclusion was not robust.

\section{Sensitivity analysis}

We performed a sensitivity analysis by omitting each study by turns to evaluate result reliability. The corresponding pooled $\mathrm{HR}$ values for OS (Figure 4A) and DFS/PFS (Figure 4B) were not significantly altered, which suggested the reliability of our conclusions.

\section{Discussion}

It is now generally accepted that the coagulation system is often activated in cancer patients [38, 39]. And the extent of coagulation activation, as estimated by the plasma fibrinogen and D-dimer, has been correlated with invasion, progression, angiogenesis and metastasis in several tumors [8, 40-42], including lung cancer $[17,29,31]$. Moreover, several studies have reported conflicting findings on the role of high plasma fibrinogen and prognosis in lung cancer.

Therefore, we performed this meta-analysis to assess the prognostic role of high plasma fibrinogen in lung cancer patients. The results of our meta-analysis demonstrated that elevated plasma fibrinogen could significantly predict poor OS and DFS/PFS of lung cancer. Additionally, significant heterogeneity was observed in the meta-analysis. Thus, we performed subgroup analysis by grouping the eligible studies according 
to analysis of variable, ethnicity, cut-off value, tumor stage, histology, and quality score to explore the source of heterogeneity. Although the heterogeneity could not eliminate, most of the prognostic significance were not unaffected by subgroup analysis stratified by analysis of variable, ethnicity, tumor stage, histology, and quality score. Furthermore, when stratified by cut-off value for OS and DFS/PFS, there were no significant heterogeneity both in

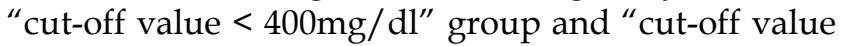
$\geq 400 \mathrm{mg} / \mathrm{dl}$ " group. So the definition of cut-off value of hyperfibrinogenemia might be the main source of heterogeneity. And the results of "cut-off value $\geq$ $400 \mathrm{mg} / \mathrm{dl}$ " group demonstrated that the high level of fibrinogen in serum was associated with worse OS and DFS/PFS of lung cancer. However, the results of another subgroup indicated that elevated serum fibrinogen was related with poor OS of lung cancer, not related to DFS/PFS. This finding suggested that hyperfibrinogenemia defined as a plasma fibrinogen concentration of $\geq 400 \mathrm{mg} / \mathrm{dl}$ could help accurately predict the prognosis of lung cancer patients both for OS and DFS/PFS. Because of publication bias for DFS/PFS existed, the elevated plasma fibrinogen could be a promising indicator for worse OS in lung cancer patients, not for DFS/PFS. In addition, hyperfibrinogenemia might be a stratified variable in clinical trials of lung cancer.

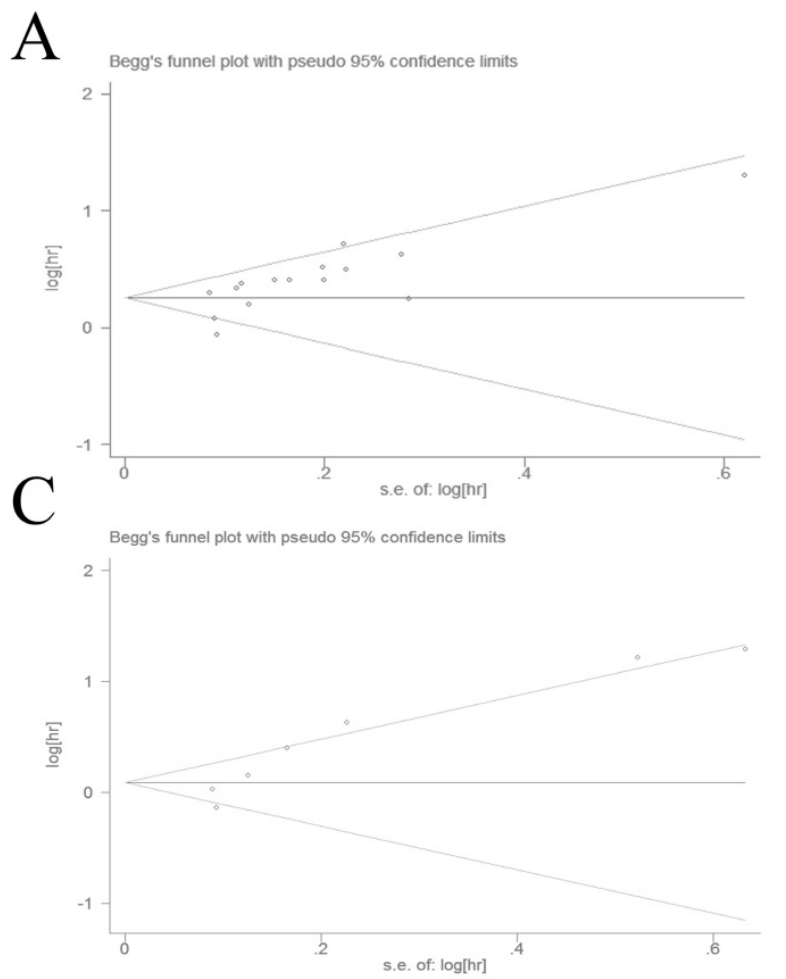

The exact reason for the association between elevated plasma fibrinogen and poor survival of patients with lung cancer remained unclear. However, several experimental studies had demonstrated that fibrinogen could play a key role in tumor progression. By promoting the stable adhesion of tumor cells and survival of metastatic emboli after tumor cells intravasation, fibrinogen had been shown to play an important determinant of spontaneous metastatic potential in lung and skin cancer models $[43,44]$. In addition, study found that fibrinogen could promote gallbladder cancer cells EMT through increasing the expression of vimentin and decreasing the expression of E-cadherin, thereby enhanced the migration and metastasis of tumor cells [45]. Moreover, with the help of thrombin and platelets, fibrinogen formed dense layers of fibrinogen that adhered to the surrounding of tumor cells and protected them from being killed by natural-killer cells [46]. Furthermore, some reports suggested that tumor cells were capable of endogenous production of fibrinogen, and that fibrinogen binding to FGF-2 and VEGF could potentiate stimulation of endothelial cell proliferation, resulting in augmented angiogenesis [47-49]. As fibrinogen could promote tumor migration, metastasis and angiogenesis, it could become a prognostic factor of lung cancer.
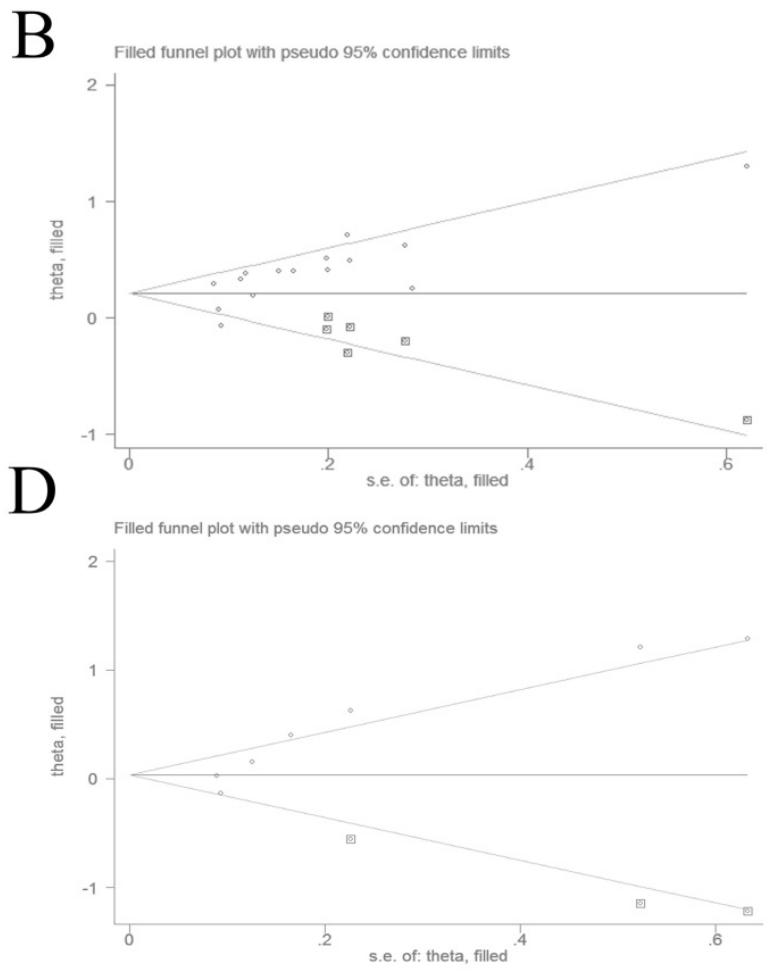

Figure 3. Begg's funnel plots evaluating possible publication bias for (A) OS without trim and fill method; (B) OS with trim and fill method; (C) DFS/PFS without trim and fill method; (D) DFS/PFS with trim and fill method. 
Recently, a number of meta-analysis had reported the prognostic role of elevated plasma fibrinogen in a variety of tumors, including, epithelial ovarian cancer [50], Renal Cell Carcinoma [51], esophageal cancer [52]. To the best of our knowledge, this meta-analysis was the first study to systematically assess the prognostic value of plasma fibrinogen in lung cancer. The results of our meta-analysis on the prognostic role of plasma fibrinogen in lung cancer were in accordance with the prognostic value of plasma fibrinogen in other tumors, indicating that high plasma fibrinogen could be widely used as a marker for poor prognosis in solid tumors, which was consistent with the results from Perisanidis's study [13]. Since the important prognostic value of plasma fibrinogen in various malignant tumors, we speculate that plasma fibrinogen can be combined with traditional clinicpathological prognostic parameters,

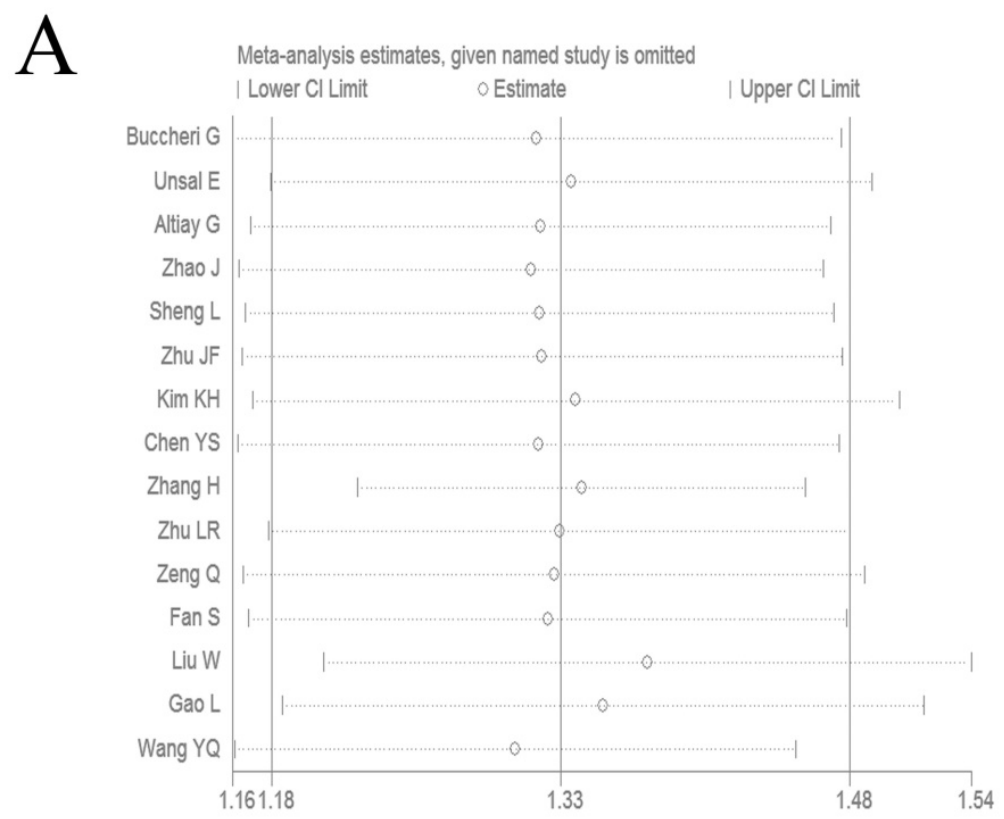

B

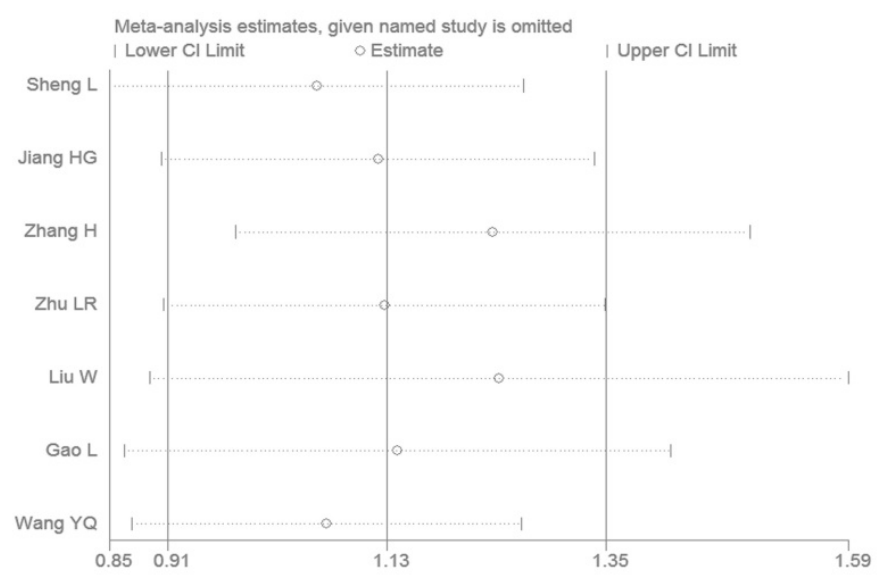

Figure 4. Sensitivity analysis on the relationship between elevated plasma fibrinogen and OS (A) or DFS/PFS (B) in lung cancer patients. which improve the treatment decision and prognosis evaluation more effectively. And plasma fibrinogen is easily available because it is a conventional, inexpensive and simple repeatable inspection result. Therefore, plasma fibrinogen can be easily integrated into daily clinical use.

In addition to the inherent deficiencies associated with meta-analysis, our study had several other limitations. Firstly, although 16 studies including 6881 lung cancer patients were included in our meta-analysis, most of the patients enrolled came from Asian region, and only 3 studies including 383 patients came from non-Asian regions, which might limit the suitable applicability of our research results to other populations. Secondly, only two studies investigated prognostic role of high plasma fibrinogen in SCLC, so further larger sample studies were needed to confirm the conclusion. Thirdly, our meta-analysis only contained articles published in English, which might lead to an inevitable bias. Fourthly, in spite of no significant publication bias for OS was observed based on the trim and fill method, we could not avoid it completely. Because positive results of studies tended to be accepted by journals easily, while negative results were likely to be rejected or even unpublished. Finally, the studies included in our meta-analysis were retrospective, observational studies, and no prospective cohort study was selected.

\section{Conclusions}

In conclusion, our meta-analysis revealed that elevated plasma fibrinogen, particularly defined as a plasma fibrinogen concentration of $\geq 400 \mathrm{mg} / \mathrm{dl}$, could be a promising indicator for worse OS in lung cancer patients, including NSCLC. And the prognostic value of plasma fibrinogen for DFS/PFS in lung cancer needed further confirmation. However, since the limitations listed above, better-designed studies on larger sample and other ethnic groups are required to further confirm our conclusion.

\section{Abbreviations}

HR: hazard ratio; $\mathrm{CI}$ : confidence interval; NSCLC: non-small cell lung cancer; SCLC: small cell lung cancer; NR: not reported; OS: overall survival; DFS: disease-free survival; PFS: progress-free survival; $\mathrm{U}$ : univariate analysis; $\mathrm{M}$ : multivariate analysis. 


\section{Acknowledgments}

This study was supported by Jiangsu Provincial Special Program of Medicine Science (No BE2015611).

\section{Competing Interests}

The authors have declared that no competing interest exists.

\section{References}

1. Siegel RL, Miller KD, Jemal A. Cancer statistics, 2016. CA Cancer J Clin. 2016; 66(1): 7-30.

2. Siegel RL, Miller KD, Jemal A. Cancer statistics, 2015. CA Cancer J Clin. 2015; 65(1): 5-29.

3. Cedrés S, Nuñez I, Longo M, et al. Serum tumor markers CEA, CYFRA21-1, and CA-125 are associated with worse prognosis in advanced non-small-cell lung cancer (NSCLC). Clin Lung Cancer. 2011; 12(3): 172-179.

4. Barlési F, Gimenez C, Torre JP, et al. Prognostic value of combination of Cyfra 21-1, CEA and NSE in patients with advanced non-small cell lung cancer. Respir Med. 2004; 98(4): 357-362.

5. Li J, Dai CH, Chen P, et al. Survival and prognostic factors in small cell lung cancer. Med Oncol. 2010; 27(1): 73-81.

6. Bremnes RM, Sundstrom S, Aasebø U, et al. The value of prognostic factors in small cell lung cancer: results from a randomised multicenter study with minimum 5 year follow-up. Lung Cancer. 2003; 39(3): 303-313.

7. Hanagiri T, Sugaya M, Takenaka M, et al. Preoperative CYFRA 21-1 and CEA as prognostic factors in patients with stage I non-small cell lung cancer. Lung Cancer. 2011; 74(1): 112-117.

8. Tsimafeyeu IV, Demidov LV, Madzhuga AV, et al. Hypercoagulability as a prognostic factor for survival in patients with metastatic renal cell carcinoma. J Exp Clin Cancer Res. 2009; 28: 30.

9. Ay C, Dunkler D, Pirker R, et al. High D-dimer levels are associated with poor prognosis in cancer patients. Haematologica. 2012; 97(8): 1158-1164.

10. Palumbo JS. Mechanisms linking tumor cell-associated procoagulant function to tumor dissemination. Semin Thromb Hemost. 2008; 34(2): 154-160.

11. Kvolik S, Jukic M, Matijevic M, et al. An overview of coagulation disorders in cancer patients. Surg Oncol. 2010; 19(1): e33-46.

12. Im JH, Fu W, Wang $\mathrm{H}$, et al. Coagulation facilitates tumor cell spreading in the pulmonary vasculature during early metastatic colony formation. Cancer Res. 2004; 64(23): 8613-8619.

13. Perisanidis C, Psyrri A, Cohen EE, et al. Prognostic role of pretreatment plasma fibrinogen in patients with solid tumors: A systematic review and meta-analysis. Cancer Treat Rev. 2015; 41(10): 960-970.

14. Unsal E, Atalay F, Atikcan S, et al. Prognostic significance of hemostatic parameters in patients with lung cancer. Respir Med. 2004; 98(2): 93-98.

15. Gao L, Zhang H, Zhang B, et al. Prognostic value of combination of preoperative platelet count and mean platelet volume in patients with resectable non-small cell lung cancer. Oncotarget. 2017; 8(9): 15632-15641.

16. Liu W, Ha M, Yin N. Combination of platelet count and lymphocyte to monocyte ratio is a prognostic factor in patients undergoing surgery for non-small cell lung cancer. Oncotarget. 2017; 8(42): 73198-73207.

17. Fan S, Guan Y, Zhao G, et al. Association between plasma fibrinogen and survival in patients with small-cell lung carcinoma. Thorac Cancer. 2017; 9(1): 146-151.

18. Stang A. Critical evaluation of the Newcastle-Ottawa scale for the assessment of the quality of nonrandomized studies in meta-analyses. Eur J Epidemiol. 2010; 25(9): 603-605.

19. Higgins JP, Thompson SG, Deeks JJ, et al. Measuring inconsistency in meta-analyses. BMJ. 2003; 327(7414): 557-560

20. Kontopantelis E, Reeves D. Performance of statistical methods for meta-analysis when true study effects are non-normally distributed: A simulation study. Stat Methods Med Res. 2012; 21(4): 409-426.

21. Brockwell SE, Gordon IR. A comparison of statistical methods for meta-analysis. Stat Med. 2001; 20(6): 825-840.

22. Egger M, Davey SG, Schneider M, et al. Bias in meta-analysis detected by a simple, graphical test. BMJ. 1997; 315(7109): 629-634.

23. Begg CB, Mazumdar M. Operating characteristics of a rank correlation test for publication bias. Biometrics. 1994; 50(4): 1088-1101.

24. Wang J, Sheng Z, Yang W, et al. Elevated Serum Concentration of Chitinase 3-Like 1 is an Independent Prognostic Biomarker for Poor Survival in Lung Cancer Patients. Cell Physiol Biochem. 2016; 38(2): 461-468.

25. Buccheri G, Ferrigno D, Ginardi C, et al. Haemostatic abnormalities in lung cancer: prognostic implications. Eur J Cancer. 1997; 33(1): 50-55.

26. Altiay G, Ciftci A, Demir M, et al. High plasma D-dimer level is associated with decreased survival in patients with lung cancer. Clin Oncol ( $\mathrm{R}$ Coll Radiol). 2007; 19(7): 494-498.

27. Zhao J, Zhao M, Jin B, et al. Tumor response and survival in patients with advanced non-small-cell lung cancer: the predictive value of chemotherapy-induced changes in fibrinogen. BMC Cancer. 2012; 12: 330.
28. Sheng L, Luo $M$, Sun $X$, et al. Serum fibrinogen is an independent prognostic factor in operable nonsmall cell lung cancer. Int J Cancer. 2013; 133(11): 2720-2725.

29. Zhu JF, Cai L, Zhang XW, et al. High plasma fibrinogen concentration and platelet count unfavorably impact survival in non-small cell lung cancer patients with brain metastases. Chin J Cancer. 2014; 33(2): 96-104.

30. Kim KH, Park TY, Lee JY, et al. Prognostic significance of initial platelet counts and fibrinogen level in advanced non-small cell lung cancer. J Korean Med Sci. 2014; 29(4): 507-511.

31. Jiang HG, Li J, Shi SB, et al. Value of fibrinogen and D-dimer in predicting recurrence and metastasis after radical surgery for non-small cell lung cancer. Med Oncol. 2014; 31(7): 22

32. Chen $\mathrm{Y}$, Zeng D, Li H, et al. Clinical and prognostic significance of plasma fibrinogen in lung cancer. J Cancer Res Ther. 2014; 2: 14-21.

33. Zhang H, Zhang L, Zhu K, et al. Prognostic Significance of Combination of Preoperative Platelet Count and Neutrophil-Lymphocyte Ratio (COP-NLR) in Patients with Non-Small Cell Lung Cancer: Based on a Large Cohort Study. PLoS One. 2015; 10(5): e0126496.

34. Zhu LR, Li J, Chen P, et al. Clinical significance of plasma fibrinogen and D-dimer in predicting the chemotherapy efficacy and prognosis for small cell lung cancer patients. Clin Transl Oncol. 2016; 18(2): 178-188.

35. Zeng Q, Xue N, Dai D, et al. A Nomogram based on Inflammatory Factors C-Reactive Protein and Fibrinogen to Predict the Prognostic Value in Patients with Resected Non-Small Cell Lung Cancer. J Cancer. 2017; 8(5): 744-753.

36. Wang YQ, Zhi QJ, Wang XY, et al. Prognostic value of combined platelet, fibrinogen, neutrophil to lymphocyte ratio and platelet to lymphocyte ratio in patients with lung adenosquamous cancer. Oncol Lett. 2017; 14(4): 4331-4338.

37. Parmar MK, Torri V, Stewart L. Extracting summary statistics to perform meta-analyses of the published literature for survival endpoints. Stat Med. 1998; 17(24): 2815-2834.

38. Grivennikov SI, Greten FR, Karin M. Immunity, inflammation, and cancer. Cell. 2010; 140(6): 883-899.

39. McMillan DC. The systemic inflammation-based Glasgow Prognostic Score: a decade of experience in patients with cancer. Cancer Treat Rev. 2013; 39(5): 534-540.

40. Batschauer AP, Figueiredo CP, Bueno EC, et al. D-dimer as a possible prognostic marker of operable hormone receptor-negative breast cancer. Ann Oncol. 2010; 21(6): 1267-1272.

41. Khoury JD, Adcock DM, Chan F, et al. Increases in quantitative D-dimer levels correlate with progressive disease better than circulating tumor cell counts in patients with refractory prostate cancer. Am J Clin Pathol. 2010; 134(6): 964-969.

42. Diao D, Zhu K, Wang Z, et al. Prognostic value of the D-dimer test in oesophageal cancer during the perioperative period. J Surg Oncol. 2013; 108(1): 34-41.

43. Palumbo JS, Kombrinck KW, Drew AF, et al. Fibrinogen is an important determinant of the metastatic potential of circulating tumor cells. Blood. 2000; 96(10): 3302-3309.

44. Palumbo JS, Potter JM, Kaplan LS, et al. Spontaneous hematogenous and lymphatic metastasis, but not primary tumor growth or angiogenesis, is diminished in fibrinogen-deficient mice. Cancer Res. 2002; 62(23): 6966-6972.

45. Shu YJ, Weng $H$, Bao RF, et al. Clinical and prognostic significance of preoperative plasma hyperfibrinogenemia in gallbladder cancer patients following surgical resection: a retrospective and in vitro study. BMC Cancer. 2014; 14: 566.

46. Zheng S, Shen J, Jiao $Y$, et al. Platelets and fibrinogen facilitate each other in protecting tumor cells from natural killer cytotoxicity. Cancer Sci. 2009; 100(5): 859-865

47. Sahni A, Simpson-Haidaris PJ, Sahni SK, et al. Fibrinogen synthesized by cancer cells augments the proliferative effect of fibroblast growth factor-2 (FGF-2). J Thromb Haemost. 2008; 6(1): 176-183.

48. Sahni A, Khorana AA, Baggs RB, et al. FGF-2 binding to fibrin(ogen) is required for augmented angiogenesis. Blood. 2006; 107(1): 126-131.

49. Sahni A, Francis CW. Vascular endothelial growth factor binds to fibrinogen and fibrin and stimulates endothelial cell proliferation. Blood. 2000; 96(12): 3772-3778

50. Luo Y, Kim HS, Kim M, et al. Elevated plasma fibrinogen levels and prognosis of epithelial ovarian cancer: a cohort study and meta-analysis. J Gynecol Oncol. 2017; 28(3): e36.

51. Tian Y, Hong M, Jing S, et al. Clinical and Prognostic Effect of Plasma Fibrinogen in Renal Cell Carcinoma: A Meta-Analysis. Biomed Res Int. 2017; 2017: 9591506.

52. Liu FT, Gao H, Wu CW, et al. The association of plasma fibrinogen with clinicopathological features and prognosis in esophageal cancer patients. Oncotarget. 2017; 8(54): 93029-93038. 